\title{
ParticipACTION, cinq ans après sa relance : enquête quantitative sur son rayonnement et sur le pouvoir d'action des organisations au Canada en matière d'initiatives consacrées à l'activité physique
}

\author{
Guy Faulkner, Ph. D. (1); Subha Ramanathan, Ph. D. (1); Ronald C. Plotnikoff, Ph. D. (2); Tanya Berry, Ph. D. (3); \\ Sameer Deshpande, Ph. D. (4); Amy E. Latimer-Cheung, Ph. D. (5); Ryan E. Rhodes, Ph. D. (6); \\ Mark S. Tremblay, Ph. D. (7); John C. Spence, Ph. D. (3)
}

Cet article de recherche quantitative originale a fait l'objet d'une évaluation par les pairs.

Diffuser cet article sur Twitter

\section{Résumé}

Introduction. ParticipACTION est un organisme canadien relancé en 2007 dédié à la communication et au marketing social en matière d'activité physique. Cette étude porte sur le pouvoir d'action des organismes canadiens dans l'adoption, la mise en œuvre et la promotion des initiatives en ce domaine. Nos objectifs étaient de comparer les résultats de base (2008) avec les résultats de suivi (2013) en ce qui concerne (1) la connaissance de ParticipACTION, (2) la capacité d'un organisme à adopter, à mettre en œuvre et à promouvoir des initiatives d'activité physique et (3) les différences potentielles en matière de pouvoir d'action d'une organisation en fonction de sa taille, de son secteur et de son mandat ainsi que (4) d'évaluer la perception de ParticipACTION cinq ans après sa relance.

Méthodologie. Dans le cadre de cette étude transversale, des représentants d'organismes locaux, provinciaux, territoriaux et nationaux ont rempli un questionnaire en ligne visant à évaluer la capacité de leur organisme à adopter, à mettre en œuvre et à promouvoir des initiatives d'activité physique. Nous avons utilisé des méthodes de statistique descriptive et des analyses de variance à un facteur pour répondre à nos objectifs.

Résultats. Le taux de réponse correspondant aux personnes ayant ouvert le courriel d'invitation à répondre au sondage et ayant consenti à y participer était de 40,6\% (685/1 688). Au total, 540 questionnaires de sondage ont été remplis. Le taux de connaissance de ParticipACTION, qui se chiffrait à 54,6\% lors de l'étude initiale, avait atteint 93,9 \% au moment du suivi (objectif 1 ). Tant les résultats initiaux que les résultats de suivi ont fait état d'un pouvoir d'action important des organismes dans l'adoption, la mise en œuvre et la promotion d'initiatives d'activité physique (objectif 2), avec cependant de légères variations en fonction du secteur et du mandat de chaque organisme (objectif 3). La plupart des répondants ont affirmé que ParticipACTION exerçait un leadership positif $(65,3 \%)$, mais les avis étaient plus partagés quant à son rôle de conseil aux infrastructures $(44,0 \%)$ ou encore de soutien à la motivation organisationnelle (47,1\%) (objectif 4).

Conclusion. Les organismes canadiens font marque d'un pouvoir d'action important en matière d'adoption, de mise en œuvre et de promotion d'initiatives d'activité physique. Cependant, cinq ans après la relance de ParticipACTION et malgré une meilleure reconnaissance de sa contribution globale au secteur de l'activité physique, aucun changement notable n'a été relevé dans les indicateurs mesurant le pouvoir d'action des organismes.

Mots-clés : activité physique, promotion de la santé, pouvoir d'action des organisations
Points saillants

- Les organismes canadiens dans le domaine de l'activité physique font marque d'un pouvoir d'action important dans l'adoption, la mise en œuvre et la promotion d'initiatives d'activité physique.

- Cinq ans après la relance de ParticipACTION, aucun changement notable n'a été relevé dans les dimensions caractérisant le pouvoir d'action des organisations.

- La majorité des répondants étaient d'accord ou tout à fait d'accord pour dire que ParticipACTION avait contribué positivement au secteur de l'activité physique et du sport et à ce que la population canadienne soit plus active.

\section{Introduction}

Le développement de la capacité des organismes à promouvoir et à mettre en œuvre des initiatives d'activité physique constitue un maillon essentiel dans la lutte contre l'inactivité physique à l'échelle de la population. Selon l'Organisation mondiale de la santé (OMS), le renforcement de ce pouvoir d'action se définit comme un processus qui vise à se doter des connaissances, des compétences, des engagements, des structures, des systèmes et du leadership

Rattachement des auteurs :

1. École de kinésiologie, Université de la Colombie-Britannique, Vancouver (Colombie-Britannique), Canada

2. School of Education, Université de Newcastle, Newcastle (Nouvelle-Galles du Sud), Australie

3. Faculté de kinésiologie, des sports et des loisirs, Université de l'Alberta, Edmonton (Alberta), Canada

4. Faculté de gestion, Université de Lethbridge, Lethbridge (Alberta), Canada

5. École de kinésiologie et d'études sur la santé, Université Queen's, Kingston (Ontario), Canada

6. École des sciences de l'exercice, de l'éducation physique et de l'éducation sur la santé, Université de Victoria, Victoria (Colombie-Britannique), Canada

7. Groupe de recherche sur les saines habitudes de vie et l'obésité, Institut de recherche du Centre hospitalier pour enfants de l'est de l'Ontario, Ottawa (Ontario), Canada

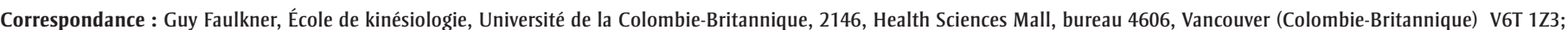
tél. : 604-822-2990; téléc. : 604-822-6842; courriel : guy.faulkner@ubc.ca 
nécessaires pour assurer une promotion efficace de la santé1, p. ${ }^{341}$. C'est donc une composante " en amont » essentielle à la promotion de la santé, qui fournit aux personnes les connaissances et les compétences requises pour favoriser l'activité physique et fait ainsi en sorte que leur organisme dispose des ressources et des infrastructures nécessaires pour soutenir des initiatives, établir et maintenir des partenariats et assurer le leadership et l'orientation du travail en vue de l'atteinte d'objectifs communs. À première vue, il est difficile de déterminer comment s'attaquer efficacement à la prévalence élevée de l'inactivité physique au Canada ${ }^{2,3}$ sans un pouvoir d'action suffisante ${ }^{4}$. Or l'évaluation des initiatives menées à l'échelle de la population porte généralement sur les changements de comportement des personnes (p. ex. comportement lié à l'activité physique) plutôt que sur les facteurs systémiques distaux (p. ex. pouvoir d'action d'une organisation) qui sous-tendent ces changements de comportement ${ }^{5}$.

Lorsque ParticipACTION a été relancé en 2007, sa mission a été élargie, au delà de la mise en œuvre de programmes d'activité physique, à des activités de marketing social, de communication et de synergie des partenariats au Canada ${ }^{6}$, en particulier dans le but d'encourager et de soutenir des actions coordonnées de la part des divers organismes et de contribuer au renforcement des compétences des collectivités. L'amélioration de la capacité des organismes canadiens à assurer une bonne mobilisation et à promouvoir l'activité physique a été ainsi définie comme l'un des principaux objectifs de ParticipACTION. Dans ce but, nous avons recueilli en 2007 des données sur la connaissance de ParticipACTION par divers organismes et sur leurs perceptions à pouvoir mobiliser et promouvoir l'activité physique ${ }^{7}$. Il s'agissait là d'une occasion exceptionnelle de suivre et d'évaluer de façon continue, d'une part, l'influence de ParticipACTION sur le pouvoir d'action d'une organisation et, d'autre part, la capacité des divers organismes à mettre en œuvre les initiatives de ParticipACTION. Les éléments clés en matière de pouvoir d'action d'une organisation sont la mise sur pied de coalitions, le réseautage, la planification, la gestion, l'exécution et l'évaluation de programmes, l'acquisition de ressources et la disponibilité de ces ressources aux fins de promotion de l'activité physique. Cependant, nos connaissances sur le pouvoir d'action d'une organisation et sur le développement de ce dernier sont limitées ${ }^{8}$.

Au départ, nous voulions comprendre comment ParticipACTION pouvait constituer pour les organismes à la fois un stimulus et une ressource en matière de développement de leur pouvoir d'action, que ce soit sur le plan de leur leadership (p. ex. processus de création de partenariats, de relations de collaboration et de liens au sein de la collectivité [voir?]), sur le plan de l'élaboration de politiques ou de " volonté » (p. ex. processus de mise en place, dans une collectivité donnée, d'une vision, d'une mission et d'une volonté politique destinées à mettre en œuvre une initiative en matière de santé et à la maintenir) ou encore sur le plan des infrastructures (p. ex. processus d'élaboration d'une organisation et d'un système de soutien dans le secteur de la santé, ou encore compétences, connaissances et ressources en matière de promotion de la santé) ${ }^{10}$. Nous nous attendions à ce que le pouvoir d'action d'un organisme du point de vue du leadership, de la volonté et des infrastructures influe sur sa capacité à adopter et à mettre en œuvre les initiatives de ParticipACTION ${ }^{11}$.

Nous avons recueilli des données avant que ParticipACTION commence à diffuser de l'information (données initiales). À l'aide d'un sondage en ligne, des intervenants clés canadiens ( $\mathrm{n}=268$; taux de réponse $=29,7 \%$ ) représentatifs d'organismes provinciaux et nationaux de divers secteurs (p. ex. sports, loisirs, santé publique, éducation) ont répondu à des questions sur la connaissance de ParticipACTION au sein de leur organisme et sur son pouvoir d'action en matière de promotion de l'activité physique ${ }^{11}$. Les résultats fondés sur l'autodéclaration ont fait état d'un pouvoir d'action des organismes canadiens important en matière de promotion de l'activité physique, comme en témoigne la moyenne d'environ 4,0 (sur une échelle à 5 points, où 1 correspond à " pas du tout », et 5 , à " beaucoup ») pour ce qui a trait à la capacité à adopter, à mettre en œuvre et à promouvoir des campagnes d'activité physique $^{11}$. Aucune tendance particulière n'a été observée, mais certaines différences ont été relevées en fonction des secteurs et des mandats des organismes. Par exemple, les organismes gouvernementaux ont fait état d'une plus grande capacité à adopter de nouvelles campagnes que les organismes sans but lucratif. Les organismes à mandat éducatif ont fait état d'une capacité d'adoption supérieure à celle des organismes à mandat relevant de la catégorie " santé publique et soins de santé » et ont fait également état d'une plus grande capacité à mettre en œuvre des initiatives d'activité physique que les organismes dont le mandat relevait des sports ou des loisirs. Cette étude initiale a permis de conclure que la majorité des organismes canadiens interrogés ont dit pouvoir participer aux initiatives proposées par ParticipACTION, et ce, quels que soient leur taille, leur secteur ou leur mandat.

Des études menées précédemment dans le cadre d'initiatives de promotion de la santé au Canada ont utilisé, pour étudier les changements en matière de pouvoir d'action des organisations, des périodes de cinq ans ${ }^{10,12,13}$. Bien qu'il faille peut-être plus de cinq ans pour que certains changements en la matière interviennent dans un organisme, nous avons décidé de reprendre, cinq ans plus tard, le cadre, les procédures et les mesures d'échantillonnage utilisés dans l'étude initiale ${ }^{11}$. Les objectifs de cette nouvelle étude ont donc été de comparer les résultats initiaux (2008) avec les résultats de suivi (2013) en ce qui concerne (1) la connaissance de ParticipACTION au sein des organismes du secteur de l'activité physique, (2) leur capacité à adopter, à mettre en œuvre et à promouvoir des initiatives d'activité physique et (3) les différences possibles de pouvoir d'action de l'organisme en fonction de sa taille, de son secteur et de son mandat principal, puis (4) d'évaluer la perception de ParticipACTION cinq années après sa relance.

\section{Méthodologie}

Nous avons fait parvenir par courriel des invitations à participer à l'étude, avec jusqu'à trois messages de rappel, à l'aide d'une méthode de Dillman modifiée ${ }^{14}$. Nous avons utilisé le service de marketing par courriel Mail Chimp ${ }^{15}$ pour l'envoi des messages électroniques, le suivi du nombre de messages ouverts et du nombre de messages non distribuables (renvoyés à l'expéditeur) et pour offrir aux destinataires la possibilité de se désabonner des communications futures ${ }^{16}$. Les messages de rappel générés par le service n'ont été envoyés qu'aux personnes qui n'avaient pas cliqué sur le lien vers le sondage. Les invitations ont été transmises aux personnesressources de l'étude de 2008 (répondants et non-répondants), aux représentants des 
principales organisations provinciales qui participaient à une activité de transport scolaire actif et aux membres du réseau de partenaires de ParticipACTION (réseau virtuel d'organismes canadiens). Des échantillons transversaux indépendants ont été utilisés dans l'étude de base et dans l'étude de suivi car suivre les organismes entre les deux études n'a pas été possible. Nous avons modifié notre protocole de base : avant de faire parvenir l'invitation à répondre au sondage, nous avons également envoyé aux destinataires un courriel contenant un aperçu des objectifs du sondage ainsi que les délais de réponse ${ }^{17}$. En outre, nous avons diffusé des annonces à plus grande échelle (entre novembre et décembre 2012) par l'entremise de ParticipACTION et du réseau de partenaires de ParticipACTION pour informer les intervenants du secteur de l'activité physique que le sondage aurait lieu en janvier et février 2013. Nous avons transmis aux personnes que nous pensions être des personnes-ressources clés connaissant bien leur organisme (p. ex. directeurs ou coordonnateurs de programme) des invitations ciblées accompagnées de la mention suivante : "le questionnaire de sondage doit être rempli par un représentant de l'organisme qui connaît bien celle-ci afin que nous puissions obtenir l'information la plus exacte possible » [traduction]. Au moment d'accéder au questionnaire de sondage, il était possible de donner son consentement, de commencer à répondre au sondage ou de quitter la page. Les répondants étaient invités à la fin du sondage à participer à une étude qualitative de suivi. Au total, 1688 répondants ont ouvert le courriel contenant le lien vers le sondage. Les détails sur le calcul des taux d'efficacité sont disponibles ailleurs ${ }^{16}$. L'étude a reçu l'approbation éthique du Comité d'éthique de la recherche de l'Université de Toronto.

\section{Mesures}

Notre outil en ligne était une version modifiée du questionnaire initial créé dans les deux langues officielles (français et anglais) à l'aide de Survey Monkey. À l'origine, le questionnaire avait été élaboré par des membres de l'équipe de recherche, puis des examinateurs externes en avaient évalué la conception pour s'assurer qu'il était complet et d'un accès et une navigation faciles. Nous avons utilisé les mêmes mesures, en y ajoutant des éléments concernant la perception de l'influence de ParticipACTION. Les caractéristiques liées à l'organisme étaient sa taille (moins de
10 employés, de 10 à 39 employés, 40 employés ou plus), le nombre d'années investies dans le domaine de l'activité physique ou de la promotion des soins de santé, la portée des activités (locale, provinciale, nationale), le secteur (gouvernement, sans but lucratif, secteur privé) et le mandat principal (santé publique ou soins de santé, sports ou loisirs, éducation). Pour chacune des questions du sondage, les répondants pouvaient passer à la question suivante sans répondre à la question en cours.

\section{Connaissance de ParticipACTION}

La connaissance de ParticipACTION a été évaluée à l'aide de questions à un seul élément : "Avez-vous entendu parler de ParticipACTION au cours des 12 derniers mois? » (réponse : oui ou non), « Connaissezvous des ressources de ParticipACTION? " (réponse : oui ou non) et «Comment avezvous entendu parler de la "nouvelle version” de ParticipACTION? " (choix de réponse : médias [journaux, télévision, radio, Internet], gouvernement, autres organisations, bouche à oreille et autre).

\section{Échelles de mesure du pouvoir d'action d'un organisme}

Le pouvoir d'action d'un organisme, ou sa capacité organisationnelle (a) à adopter une nouvelle initiative d'activité physique (7 éléments; $\alpha=0,92$ ), (b) à mettre en œuvre une nouvelle initiative d'activité physique (11 éléments; $\alpha=0,92$ ) et (c) à promouvoir à l'externe une nouvelle initiative d'activité physique (9 éléments; $\alpha=0,87)$ a été évalué au moyen de trois échelles à cinq choix de réponse, 1 correspondant à " pas du tout » et 5 à " beaucoup ". Le coefficient alpha de Cronbach $(\alpha)$ permet d'estimer la cohérence interne, c'est-à-dire le degré selon lequel les éléments d'une échelle mesurent conjointement le même concept ${ }^{18}$. Sa valeur varie entre 0 et 1 , et un coefficient supérieur à 0,70 témoigne généralement d'une bonne cohérence interne ${ }^{18}$. Les échelles employées dans notre étude sont adaptées des échelles validées élaborées pour le l'Alberta Heart Health Project (AHHP) ${ }^{10,19}$ afin d'évaluer le leadership ${ }^{20}$, les infrastructures ${ }^{7}$ et la volontée ${ }^{11}$ des organismes et qui ont fait preuve d'une bonne fiabilité au départ ${ }^{11}$.

\section{Perception de ParticipACTION}

Nous avons également évalué la perception de l'influence de ParticipACTION sur le pouvoir d'action de l'organisme à l'aide d'une échelle de Likert à 5 points, 1 correspondant à " pas du tout d'accord " et 5 à " tout à fait d'accord ». Ces nouvelles questions ont porté sur le leadership, les infrastructures et la volonté ainsi que sur la perception de l'influence globale de ParticipACTION. Les réponses " pas du tout d'accord » et " pas d'accord » ont été combinées, tout comme les réponses « tout à fait d'accord " et " d'accord ». Chaque élément a été examiné individuellement.

\section{Analyse statistique}

Pour répondre aux objectifs 1,2 et 4 (c.-à-d. pour évaluer la connaissance de ParticipACTION, tant dans sa version initiale que dans sa nouvelle version, et pour établir les niveaux initiaux associés aux trois dimensions du pouvoir d'action d'un organisme), nous avons utilisé des statistiques descriptives. Dans le cas de l'objectif 3 (qui consistait à examiner les variations potentielles entre les trois dimensions de ce pouvoir), nous avons effectué des analyses de variance unidimensionnelles à un facteur et des tests $t$ pour échantillons indépendants afin d'évaluer les différences de résultat pour chacune des trois dimensions (adopter, mettre en œuvre et promouvoir une nouvelle initiative d'activité physique) en fonction de chacune des trois caractéristiques de l'organisation (taille, secteur et mandat). Nous avons ensuite fait des comparaisons par paire, le seuil de signification statistique étant fixé à $p<0,05$. Certains questionnaires de sondage n'ayant pas été remplis au complet et certaines données étant manquantes aléatoirement, nous avons eu recours à la méthode de suppression par liste pour gérer les données manquantes de chaque analyse.

\section{Résultats}

\section{Taux de réponse}

La figure 1 illustre le déroulement de l'étude et fournit les taux de réponse au sondage. Le taux de réponse des personnes ayant ouvert le courriel contenant au lien vers le sondage et ayant consenti à y participer a été de 40,6 \% (685 personnes ayant donné leur consentement sur 1688 répondants ayant ouvert le courriel).

\section{Caractéristiques des répondants et des organismes}

La répartition des répondants issus d'organismes exerçant leurs activités à l'échelon 
provincial, territorial et local était relativement bonne et représentative de la population canadienne (voir tableau 1). La seule exception était le Québec, où la proportion de répondants était près de deux fois inférieure à la proportion attendue si l'on se base sur la répartition de la population. Le groupe d'âge le plus important était celui des 35 à 50 ans $(238 / 532=44,7 \%)$, suivi de celui des 50 ans et plus $(183 / 532=$ $34,4 \%$ ) et de celui des moins de 35 ans $(111 / 532=20,9 \%)$. Une proportion importante de répondants ont dit travailler dans un domaine lié à l'activité physique ou à la promotion de la santé depuis onze ans ou plus $(295 / 488=60,5 \%)$, mais la plupart ont déclaré faire partie de leur organisme actuel depuis dix ans ou moins (316/516 = $61,2 \%$ ) (voir tableau 2).

Quant à la population ciblée par la mission de chaque organisme en matière d'activité physique, le groupe visé dans la majorité des cas était les jeunes d'âge scolaire $(479 / 541=88,5 \%)$. Bon nombre de répondants ont également mentionné les adultes $(277 / 541=51,2 \%)$, les enfants jusqu'à 4 ans $(158 / 541=29,2 \%)$, les personnes de 65 ans et plus $(180 / 541=33,3 \%)$ et les employés de leur organisme $(74 / 541=$ $13,7 \%)$.

L'échantillon de suivi dont on rend compte ici est similaire à l'échantillon initial décrit par Plotnikoff et ses collègues ${ }^{11}$, à quelques exceptions près. Il comporte un moins grand nombre d'organismes d'échelon national $(9,6 \%$ contre $29,6 \%)$ et provincial (19,3\% contre $30,6 \%)$ et un moins grand nombre d'organismes de 40 employés ou plus à temps plein $(25,0 \%$ contre $46,6 \%)$. En outre, dans l'échantillon de suivi, la proportion d'organismes dotés d'un mandat éducatif est plus faible $(32,5 \%$ contre $48,0 \%$ ) et les proportions d'organismes dont le mandat s'inscrit dans la catégorie « urbanisme ou transports » et d'organismes du secteur privé sont supérieures (respectivement $1,3 \%$ contre $0 \%$ et $4,6 \%$ contre $1,6 \%)$.

Une question (avec réponse sur une échelle de Likert à 5 points où 1 correspond à " pas du tout " et 5 à " beaucoup ») a visé à déterminer dans quelle mesure les répondants pensaient que leurs réponses étaient représentatives de leur organisme dans son ensemble. De façon générale, les répondants ont estimé bien connaître le travail réalisé par leur organisme en matière de
FIGURE 1

Arborescence de réponse

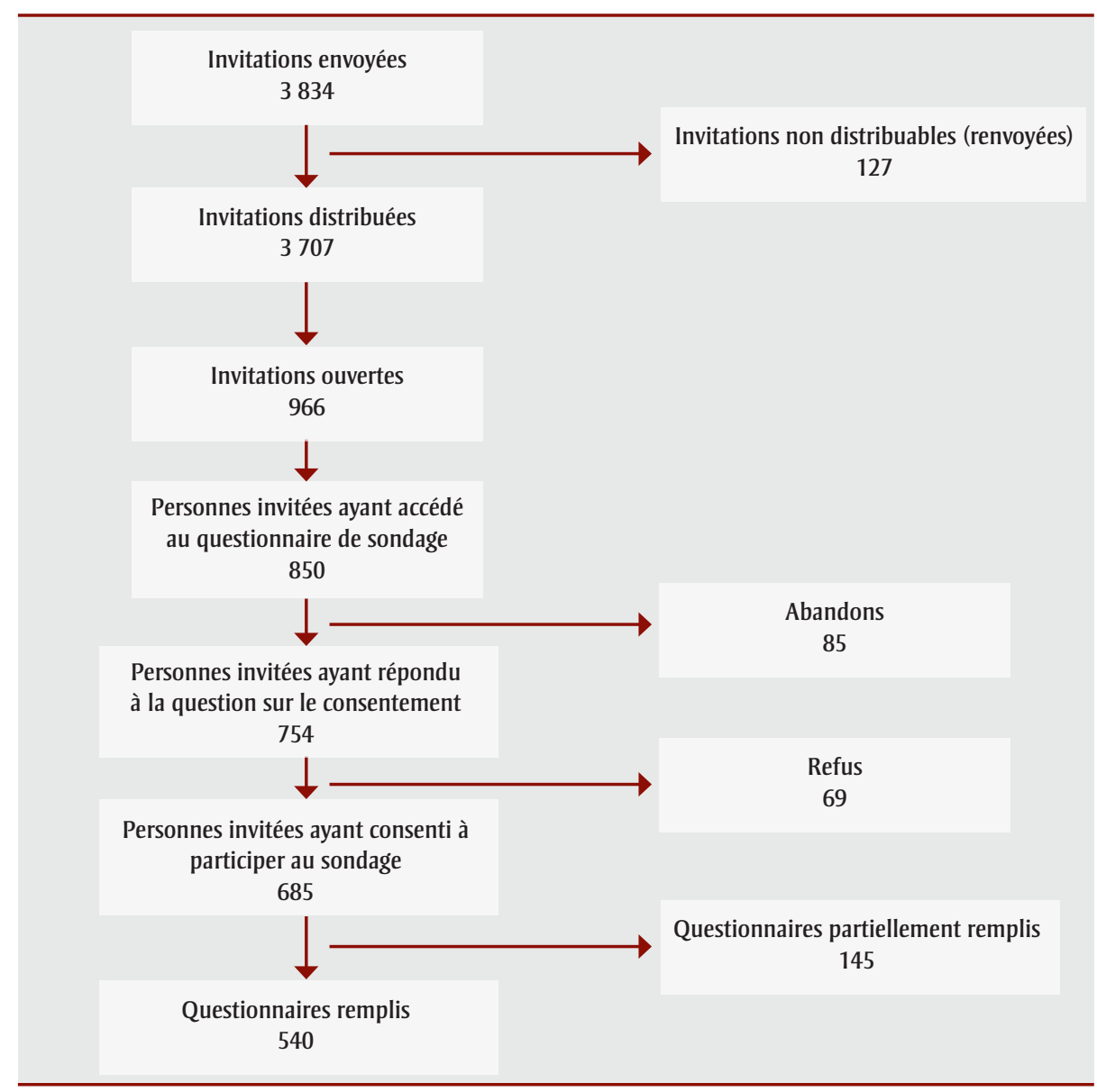

TABLEAU 1

Réponse au sondage par province ou territoire chez les répondants qui travaillent à l'échelon provincial, territorial ou local

\begin{tabular}{lrr}
\multicolumn{1}{c}{ Province ou territoire } & $\begin{array}{c}\text { Réponse de } \\
\text { l'échantillon total } \\
\mathbf{n}(\%)\end{array}$ & \% de la population canadienne \\
\hline Colombie-Britannique & $73(14,9)$ & 13,3 \\
Alberta & $67(13,6)$ & 11,1 \\
Saskatchewan & $18(3,7)$ & 3,1 \\
Manitoba & $26(5,3)$ & 3,6 \\
Ontario & $163(33,2)$ & 38,7 \\
Québec & $46(9,4)$ & 23,1 \\
Nouveau-Brunswick & $24(4,9)$ & 2,2 \\
Nouvelle-Écosse & $35(7,1)$ & 2,7 \\
île-du-Prince-Édouard & $7(1,4)$ & 0,4 \\
Terre-Neuve-et-Labrador & $17(3,5)$ & 1,5 \\
Yukon & $5(1,0)$ & 0,1 \\
Territoires du Nord-Ouest & $5(1,0)$ & 0,1 \\
Nunavut & $5(1,0)$ & 0,1 \\
Total & $491^{\mathrm{b}}(100)$ & 100 \\
\hline
\end{tabular}

a D’après les données du recensement de 2012 (Statistique Canada).

${ }^{\mathrm{b}}$ Les répondants qui travaillent à l'échelon national n'avaient pas à choisir de province ou de territoire. 
TABLEAU 2

Caractéristiques des organismes

\section{Caractéristiques}

Fréquence ${ }^{\mathrm{a}}(\mathbf{n})$

$(\%)$

Depuis combien d'années votre organisme s'investit-il dans le domaine de l'activité physique ou de la promotion de la santé?

\begin{tabular}{llrr} 
Moins de 5 ans & & 61 & 11,5 \\
De 5 à 10 ans & & 73 & 13,8 \\
De 11 à 15 ans & & 51 & 9,6 \\
De 16 à 20 ans & & 39 & 7,4 \\
Plus de 20 ans & & 305 & 57,7 \\
Total & & 529 & 100 \\
\hline Votre organisme exerce-t-il principalement ses activités à l'échelon national, provincial ou territorial, ou local'? \\
National & & 52 & 9,6 \\
Provincial ou territorial & & 105 & 19,3 \\
Local & & 348 & 64,1 \\
Régional & & 5 & 0,9 \\
Plusieurs échelons & & 24 & 4,4 \\
International & & 4 & 0,7 \\
Autre & & 5 & 0,9 \\
Total & & 543 & 100
\end{tabular}

Dans quel secteur travaillez-vous (gouvernement ou éducation, sans but lucratif ou secteur privé)?

Gouvernement ou éducation

268

49,5

Sans but lucratif

233

43,1

Secteur privé

25

4,6

Autre

15

541

1,8

Total

Dans quelle catégorie s'inscrit principalement le mandat de votre organisme?

Santé publique ou soins de santé

$\begin{array}{rr}52 & 9,6 \\ 256 & 47,3 \\ 176 & 32,5 \\ 7 & 1,3 \\ 50 & 9,2 \\ 541 & 100\end{array}$

${ }^{a}$ Les questionnaires de sondage partiellement remplis ont été inclus.

b Les répondants qui ont choisi la catégorie « autre » ont donné comme réponses « régional », « multiple » et « international ».

c Secteur : les catégories « gouvernement » et « éducation » ont été combinées, puisque tous les établissements d'enseignement sont affiliés à des commissions et à des conseils scolaires provinciaux. promotion de l'activité physique, soit $M$ (écart-type) $=4,24(0,86)$.

\section{Connaissance de ParticipACTION (objectif 1)}

Une grande majorité les 669 répondants $(93,9 \%)$ ont indiqué avoir entendu parler de ParticipACTION depuis sa relance en 2007. Dans l'échantillon initial, ce taux s'établissait à $54,6 \%$. La plupart des répondants $(502 / 626=80,2 \%)$ ont dit avoir entendu parler de ParticipACTION dans les médias (p. ex. journaux, télévision, médias sociaux). Plusieurs étaient aussi membres du réseau de ParticipACTION $(216 / 626=34,5 \%)$, participaient à un groupe consultatif ou à une initiative de ParticipACTION $(131 / 626=20,9 \%)$ ou avaient entendu parler de ParticipACTION lors d'une présentation ou d'un webinaire $(86 / 626=13,7 \%)$, par l'intermédiaire d'une liste de diffusion $(71 / 626=11,3 \%)$ ou par d'autres moyens (p. ex. organisations partenaires et collègues) $(63 / 626=$ $10,1 \%)$.

\section{Niveaux associés aux trois dimensions $d u$ pouvoir d'action de l'organisme (objectif 2)}

Le tableau 3 présente les niveaux initiaux et de suivi associés aux trois dimensions du pouvoir d'action de l'organisme (adopter, mettre en œuvre et promouvoir une nouvelle initiative d'activité physique) en fonction de sa taille, de son secteur et de son mandat (objectif 2). Comme dans l'étude de base, la moyenne s'élevait à environ 4,0 (sur une échelle à 5 points, 1 correspondant à " pas du tout » et 5 à " beaucoup ») pour chacune des dimensions et pour chacune des trois caractéristiques organisationnelles. Ces niveaux sont cohérents dans les deux études et présentent une faible variabilité, les écarts minimum et maximum entre les résultats initiaux et les résultats de suivi allant de $-0,09$ à $+0,11$.

\section{Différences en fonction de la taille, du secteur et du mandat de l'organisme (objectif 3)}

Même si l'ampleur de l'effet s'est révélée faible, nous avons observé deux différences statistiquement significatives en fonction de la taille, du secteur ou du mandat de l'organisme (objectif 3; voir tableau 3). Les organismes ne comptant aucun employé à temps plein ont déclaré avoir une capacité de mise en œuvre d'une initiative d'activité 
TABLEAU 3

Résultats moyens selon les caractéristiques des organismes dans les trois dimensions de leur pouvoir d'action, 2013 (année de suivi)

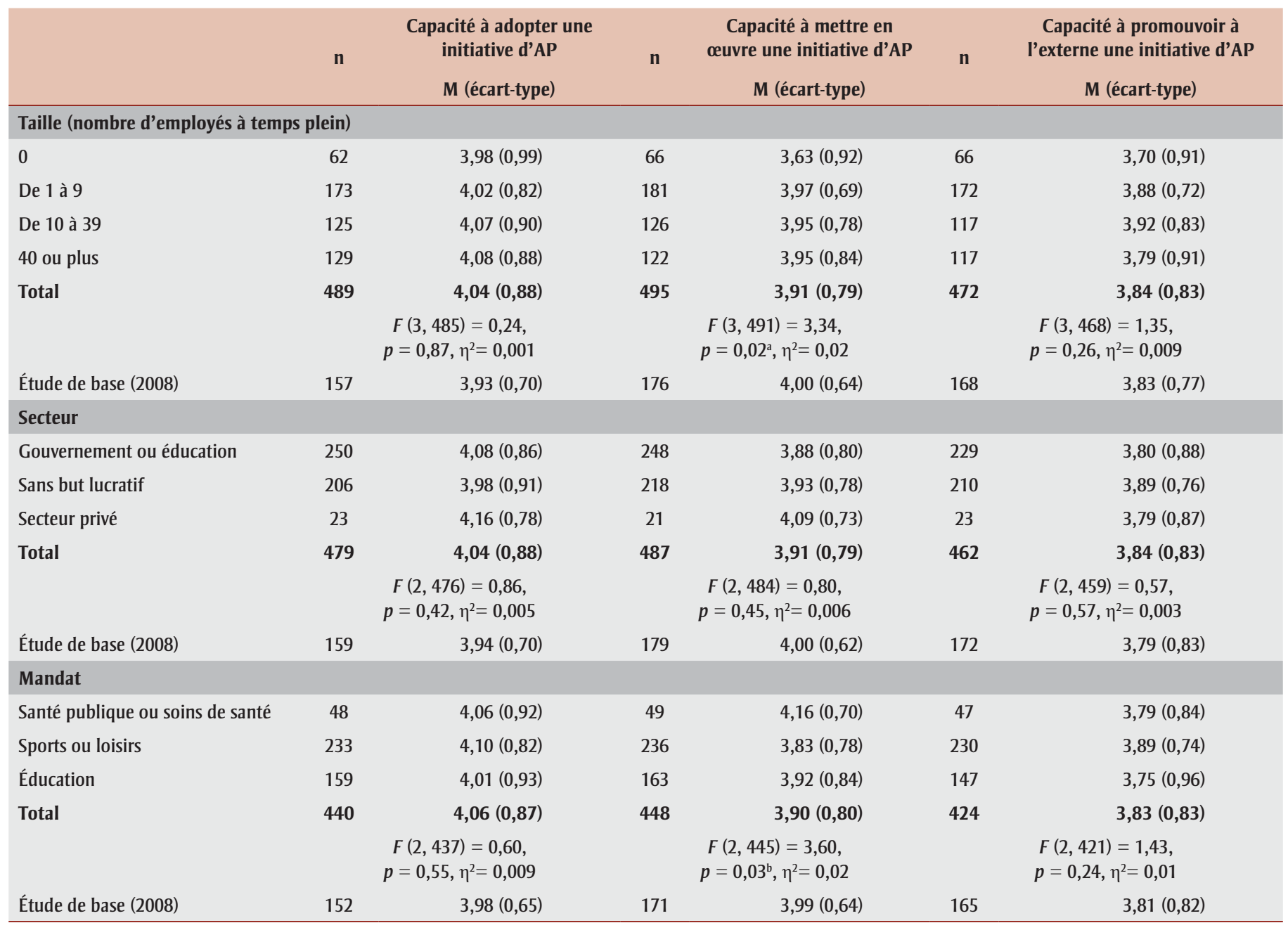

Abréviations : AP, activité physique; $M$, moyenne.

Remarques : Comme les questionnaires de sondage partiellement remplis ont été inclus, il manque certaines données.

Pour gérer les données manquantes, nous avons eu recours à la méthode de suppression par liste. Par conséquent, la valeur « $\mathbf{n}$ » varie.

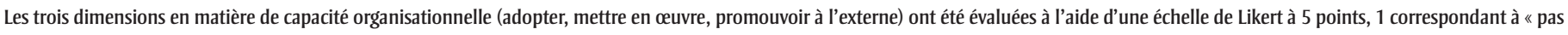
du tout » et 5 à « beaucoup ».

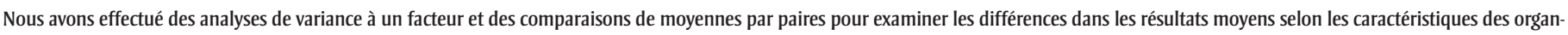
ismes pour chacune des trois dimensions en matière de capacité organisationnelle.

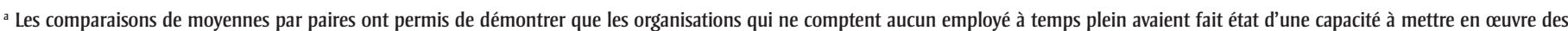
initiatives d'AP nettement inférieure à celle des organisations de toutes les autres catégories $(p<0,01)$.

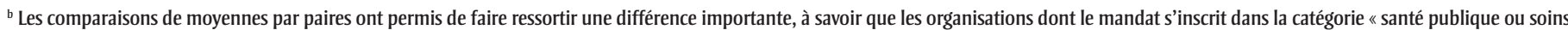
de santé » avaient fait état d'une capacité à mettre en œuvre des initiatives d'AP supérieure à celle des organisations dont le mandat s'inscrit dans la catégorie « sports ou loisirs » ( $p<0,03$ ).

physique inférieure à celle des organismes où travaillent des employés à temps plein. De plus, les organismes en santé publique ont indiqué avoir une plus grande capacité de mise en œuvre d'une initiative d'activité physique que les organismes en sports ou loisirs.

\section{Perception de ParticipACTION (objectif 4)}

La majorité des répondants étaient d'accord ou tout à fait d'accord pour dire que ParticipACTION avait contribué à rendre la population canadienne plus active (63,2\%) et apporté une contribution positive au secteur de l'activité physique et du sport $(72,9 \%)$ (voir tableau 4). Questionnés sur la contribution précise de ParticipACTION sur le plan du pouvoir d'action des organisations, la majorité des répondants ont indiqué que l'organisme avait exercé un leadership (65,3 \%), mais un moins grand nombre de répondants étaient d'accord ou tout à fait d'accord pour dire que ParticipACTION avait joué un rôle dans le pouvoir d'action et dans les infrastructures
(44 \%) ou dans la volonté ou la motivation de leur organisme $(47,1 \%)$.

\section{Analyse}

Notre étude traite de l'évolution de l'influence d'un organisme national de marketing social sur le renforcement du pouvoir d'action d'autres organismes nationaux, provinciaux et locaux. Le taux de participation, de 29,7\% (269/902) au départ, est passé à 40,6\%(685/1 688) au moment du suivi, avec deux fois plus 
TABLEAU 4

Perception de ParticipACTION

\begin{tabular}{|c|c|c|c|c|}
\hline & \multirow[t]{2}{*}{ Énoncés } & $\begin{array}{l}\text { Pas du tout } \\
\text { d'accord ou } \\
\text { pas } \\
\text { d'accord }\end{array}$ & Neutre & $\begin{array}{l}\text { Tout à fait } \\
\text { d'accord ou } \\
\text { d'accord }\end{array}$ \\
\hline & & n (\%) & n (\%) & n (\%) \\
\hline \multicolumn{5}{|c|}{$\begin{array}{l}\text { Dans quelle mesure êtes-vous d'accord ou non avec les énoncés suivants? Au cours des cinq dernières } \\
\text { années }{ }^{\mathrm{a}} . .\end{array}$} \\
\hline a) & $\begin{array}{l}\text { ParticipACTION a contribué à rendre la population } \\
\text { canadienne plus active }\end{array}$ & $55(9,2)$ & $164(27,6)$ & $376(63,2)$ \\
\hline b) & $\begin{array}{l}\text { ParticipACTION a exercé un leadership par la création } \\
\text { de partenariats, de relations de collaboration et de liens } \\
\text { avec le secteur de l'activité physique et du sport }\end{array}$ & $57(9,8)$ & $144(24,8)$ & $379(65,3)$ \\
\hline c) & $\begin{array}{l}\text { ParticipACTION a permis d'accroître la capacité } \\
\text { (connaissances, compétences et ressources) de mon } \\
\text { organisation à promouvoir l'activité physique }\end{array}$ & $171(29,1)$ & $158(26,9)$ & $259(44,0)$ \\
\hline d) & $\begin{array}{l}\text { ParticipACTION a apporté une contribution positive au } \\
\text { secteur de l'activité physique et du sport }\end{array}$ & $45(7,7)$ & $115(19,6)$ & $426(72,9)$ \\
\hline e) & $\begin{array}{l}\text { ParticipACTION a exercé un leadership par la } \\
\text { promotion de l'activité physique }\end{array}$ & $53(9,0)$ & $115(19,4)$ & $424(71,6)$ \\
\hline f) & $\begin{array}{l}\text { ParticipACTION a permis de motiver davantage mon } \\
\text { organisation à promouvoir l'activité physique au sein de } \\
\text { mon secteur }\end{array}$ & $168(29,0)$ & $139(24,0)$ & $273(47,1)$ \\
\hline
\end{tabular}

${ }^{a}$ Les catégories « pas du tout d'accord » et « pas d'accord » ont été combinées, tout comme les catégories « tout à fait d'accord » et « d'accord».

d'organismes ayant pris part à l'étude de suivi $^{16}$. Il est possible qu'un nombre accru d'organismes aient été joints dans le cadre de l'étude de suivi grâce à la facilité d'accès offerte par le réseau virtuel de partenaires de ParticipACTION et aussi du fait que de nouveaux organismes soient apparus dans le domaine de l'activité physique depuis la relance de ParticipACTION. La connaissance de ParticipACTION a augmenté, passant de 54,6 \% au moment de l'étude initiale à 93,9\% lors de l'étude de suivi, ce qui montre que la «nouvelle version » de ParticipACTION possède un bon rayonnement à l'échelle nationale. Le but de cette analyse est de fournir un aperçu du pouvoir d'action des organisations cinq ans après la relance de ParticipACTION.

Nous avons rassemblé peu d'éléments donnant à penser que la capacité à adopter, à mettre en œuvre ou à promouvoir des initiatives d'activité physique ait changé au cours des cinq dernières années, et seule une faible part des changements observés a pu être attribuée à ParticipACTION. Aux deux moments de l'étude, les organismes d'appartenance des répondants semblent avoir eu un pouvoir d'action important dans ses trois dimensions : la moyenne variait entre 3,83 et 4,10 sur une échelle à 5 points. Les résultats de base étant relativement élevés, il y a peut-être eu un effet de plafonnement ne laissant que peu de place à l'amélioration dans le cas de certains organismes. Environ 75 \% des organismes répondants ont en effet indiqué exercer leurs activités depuis plus de dix ans, ce qui fait qu'ils disposent sans doute d'une moins grande marge de manœuvre pour développer leur pouvoir d'agir. À l'opposé, pour certains organismes, en particulier ceux qui ont vu le jour récemment, il pourrait falloir plus de cinq ans avant d'être en mesure d'observer des changements en matière d'amélioration du pouvoir d'action. Le manque d'études comparables à la nôtre limite la validation de ces hypothèses. Une analyse des tendances liées au Bulletin de l'activité physique chez les jeunes de ParticipACTION a notamment révélé des changements positifs dans les stratégies et les investissements gouvernementaux et non gouvernementaux au Canada au cours des 12 dernières années $^{22}$. Ces changements transparaissent peut-être dans le pouvoir d'action important des organismes dont les participants ont fait état, du moins dans le contexte des enfants et des jeunes.

Par ailleurs, nous avons constaté que le pouvoir d'action d'un organisme variait peu en fonction de sa taille, de son secteur ou de son mandat. En fait, aucune de ces caractéristiques n'avait d'incidence sur la capacité à adopter et à promouvoir une initiative d'activité physique. Toutefois, comme nous pouvions nous y attendre, les organismes qui ne comptent aucun employé à temps plein ont fait état d'une capacité inférieure à mettre en œuvre des initiatives d'activité physique, mais les différences ne se sont pas révélées statistiquement significatives. De même, les organismes en santé publique ont fait état d'une capacité de mise en œuvre supérieure à celle des organismes en sports ou loisirs. Le mandat élargi en santé publique de ces organismes correspond peut-être davantage à celui de ParticipACTION que le mandat plus restreint des organismes en sports ou loisirs. Les différences observées étaient mineures et doivent être interprétées avec prudence.

Les répondants ont déclaré que ParticipACTION avait eu une influence sur le secteur de l'activité physique au Canada et avait contribué à rendre la population canadienne plus active, ce qui se reflète en grande partie dans la perception positive de la contribution de l'organisme sur le plan du leadership. Comme le décrivent Faulkner et ses collègues ${ }^{23}$ (voir l'introduction à ce numéro), les priorités stratégiques de ParticipACTION ont été axées sur le marketing social, la communication et l'échange de connaissances. Entre 2007 et 2012, ParticipACTION a ainsi lancé trois campagnes nationales de marketing social et a créé le réseau de partenaires de ParticipACTION pour permettre l'échange de connaissances entre organismes canadiens $^{23}$. Dans l'étude qualitative initiale, les principaux intervenants ont mentionné quelques-unes de leurs attentes à l'égard de la nouvelle version de ParticipACTION ${ }^{24}$. Ils ont entre autres souligné que, dans le cadre de son rôle de promotion, ParticipACTION devrait définir une stratégie générale en matière d'activité physique en créant une politique nationale sur l'activité physique. ParticipACTION a depuis dirigé un processus d'élaboration et de consultation approfondie en vue d'une stratégie nationale de ce type, nommée "Canada actif 20/20 » ${ }^{25}$. Toutes ces activités ont sans doute joué un rôle dans la perception des répondants quant au leadership national exercé par l'organisme. D'autres intervenants ${ }^{26}$ ont souligné à quel point faciliter les partenariats était important lorsqu'on veut renforcer le pouvoir d'action en matière de promotion de l'activité physique et du sport. Or ParticipACTION n'a fourni aucune infrastructure ni aucune 
ressource directe ou presque aux organismes canadiens, compte tenu du fait que les investissements dans les infrastructures et les ressources proviennent surtout des provinces et des territoires, mais il a plutôt accompli ce qu'il était en mesure de faire, à savoir exercer un leadership et contribuer à créer une approche unifiée de la sensibilisation à l'activité (et à l'inactivité) physique. L'étude qualitative initiale a également révélé que le degré de volonté et de motivation à participer à la promotion de l'activité physique était élevé ${ }^{4}$. Il est probable que l'influence de l'organisme sur cette dimension du pouvoir d'action soit moins apparente. D’autres études qualitatives pourraient permettre d'avoir une meilleure vue d'ensemble de la situation en approfondissant l'étude des changements potentiels en matière de pouvoir d'action des organismes, peut-être plus nuancés par nature.

\section{Limites}

Notre étude comporte trois limites importantes à ne pas négliger. Tout d'abord, sa conception transversale ne nous a pas permis d'examiner les changements survenus au fil du temps de manière aussi efficace que si nous avions eu recours à une étude longitudinale. Il y avait aussi des différences entre les deux échantillons dans la nature et la taille des organismes. On doit tenir compte de ces limites surtout lors de l'interprétation des comparaisons entre le pouvoir d'action des organismes de 2008 et celui de 2013 (objectifs 2 et 3), sachant qu'elles revêtent une importance moindre pour ce qui a trait à la connaissance (objectif 1 ) et à la perception de ParticipACTION (objectif 4). Ensuite, le taux de réponse n'a été que de 40,6 \%. Même s'il s'agit d'une amélioration par rapport au taux de l'étude initiale $(29,7 \%)$, un biais a pu être introduit dans les réponses du fait de ces deux limites. Ceci dit, l'échantillon était diversifié et nous avons employé un protocole de Dillman modifié avec messageguide préalable au sondage et plusieurs messages de rappel dans le but d'améliorer ce taux. Enfin, il n'existe aucun moyen de rendre compte des divers autres facteurs ayant une incidence sur le secteur de l'activité physique, ce qui empêche de mesurer l'influence (ou l'absence d'influence) réelle de ParticipACTION.

\section{Conclusion}

Notre étude vient enrichir le peu de documentation sur les changements en matière de pouvoir d'action des organisations. D’après nos résultats, les organismes canadiens qui s'investissent dans l'activité physique et la promotion de la santé font marque d'une bonne capacité à adopter, à mettre en œuvre et à promouvoir des initiatives d'activité physique, et ce, quels que soient leur taille, leur secteur ou leur mandat. Cinq ans après la relance de ParticipACTION, aucun changement notable n'a été relevé dans les indicateurs de ce pouvoir d'action. Toutefois, la plupart des organismes estiment que ParticipACTION contribue positivement, du point de vue du leadership, à l'activité physique et au secteur de l'activité physique en général. Les organismes connaissent très bien ParticipACTION. La réalisation de cette étude offre la preuve que surveiller les changements potentiels en matière de pouvoir d'action des organismes à l'échelle de la population est possible, et ces données peuvent servir à orienter l'évaluation continue et à long terme de l'influence de ParticipACTION.

\section{Remerciements}

Ce projet a été rendu possible grâce à une subvention de fonctionnement des Instituts de recherche en santé du Canada (MOP123491). Guy Faulkner est titulaire d'une chaire en santé publique appliquée des Instituts de recherche en santé du Canada (IRSC) et de l'Agence de la santé publique du Canada (ASPC). Amy Latimer-Cheung et Tanya R. Berry bénéficient du soutien du Programme des chaires de recherche du Canada.

\section{Conflits d'intérêts}

GF, TB, SD, AEL, RER, MST et JS sont membres du Groupe consultatif de recherche de ParticipACTION.

\section{Contribution des auteurs et avis}

GF a conceptualisé l'étude et rédigé la première ébauche de l'article. SR a dirigé la collecte et l'analyse des données. RP, TB., SD, AELC, RER, MST et JCS ont contribué à la conception de l'étude, à l'analyse et à l'interprétation des résultats ainsi qu'à la rédaction et à la révision de l'article. Tous les auteurs ont lu et approuvé la version finale du manuscrit.

Le contenu de l'article et les points de vue qui y sont exprimés n'engagent que les auteurs et ne reflètent pas nécessairement ceux du gouvernement du Canada.

\section{Références}

1. Smith BJ, Tang KC, Nutbeam D. WHO Health Promotion Glossary: new terms. Health Promot Int. 2006;21(4): 340-345. doi:10.1093/heapro/dal033.

2. Colley RC, Garriguet D, Janssen I, Craig CL, Clarke J, Tremblay MS. Activité physique des adultes au Canada : résultats d'accélérométrie de l'Enquête canadienne sur les mesures de la santé de 2007-2009. Rapports sur la santé. 2011;22(1):7-14.

3. Colley RC, Garriguet D, Janssen I, Craig CL, Clarke J, Tremblay MS. Activité physique des enfants et des jeunes au Canada : résultats d'accélérométrie de l'Enquête canadienne sur les mesures de la santé de 20072009. Rapports sur la santé. 2011;22(1): 15-23.

4. Faulkner G, McCloy C, Plotnikoff RC, et al. ParticipACTION: Baseline assessment of the capacity available to the 'New ParticipACTION': a qualitative study of Canadian organizations. Int $\mathrm{J}$ Behav Nutr Phy. 2009;6. doi: 10.1186 /1479-5868-6-87.

5. Finlay SJ, Faulkner G. Physical activity promotion through the mass media: inception, production, transmission and consumption. Prev Med. 2005; 40(2):121-130. doi: 10.1016/j.ypmed .2004.04.018

6. Latimer-Cheung A, Murmets K, Faulkner G. ParticipACTION: the national voice of physical activity and sport. Dans : Pate RR, Buchner DM (dir.). Implementing Physical Activity Strategies. Champaign, IL: Human Kinetics; 2014. p. 61-70.

7. Plotnikoff RC, Anderson D, Raine K, Cook K, Barrett L, Prodaniuk T. Scale development of individual and organization infrastructure for heart health promotion in Regional Health Authorities. Health Educ J. 2005;64:265-270. En ligne à : http://journals.sagepub .com/doi/pdf/10.1177/0017896905064 00306ble 
8. Elliott SJ, O'Loughlin J, Robinson K, Eyles J, Cameron R, Harvey D, et al. Conceptualizing dissemination research and activity: the case of the Canadian Heart Health Initiative. Health education \& behavior : the official publication of the Society for Public Health Education. 2003;30(3):267-82; discussion 83-6. doi: 10.1177/1090198103 030003003 .

9. Robinson K, Elliott SJ, Driedger SM, et al. Using linking systems to build capacity and enhance dissemination in heart health promotion: a Canadian multiple-case study. Health Educ Res. 2005;20(5):499-513. doi: 10.1093/her /cyh006.

10. Dressendorfer RH, Raine K, Dyck RJ, et al. A conceptual model of community capacity development for health promotion in the Alberta Heart Health Project. Health Promot Pract. 2005; 6(1):31-36. doi: 10.1177/152483990 3259302 .

11. Plotnikoff RC, Todosijczuk I, Faulkner G, et al. ParticipACTION: Baseline assessment of the 'new ParticipACTION': a quantitative survey of Canadian organizational awareness and capacity. Int J Behav Nutr Phy. 2009;6. doi: 10.1186/1479-5868-6-86.

12. Ebbesen LS, Heath S, Naylor PJ, Anderson D. Issues in measuring health promotion capacity in Canada: a multi-province perspective. Health Promot Int. 2004;19(1):85-94.

13. Joffres C, Heath S, Farquharson J, et al. Defining and operationalizing capacity for heart health promotion in Nova Scotia, Canada. Health Promot Int. 2004;19(1):39-49.

14. Dillman DA. Mail and telephone surveys: the Total Design Method. New York: Wiley; 1978. En ligne à : https:// www.ncbi.nlm.nih.gov/pmc/articles /PMC2328022/

15. Mail Chimp. Intégrer SurveyMonkey à MailChimp. 14 juillet 2017. En ligne à : https://kb.mailchimp.com /fr/integrations/surveys/integrate -surveymonkey-with-mailchimp

16. Ramanathan S, Faulkner G. Calculating outcome rates in web surveys. Can J Prog Eval. 2015;30(1). doi: 10.3138/cjpe.30.1.90.
17. Drummond FJ, Sharp L, Carsin A-E, Kelleher T, Comber H. Questionnaire order significantly increased response to a postal survey sent to primary care physicians. J Clin Ep. 2008;61(2):177185. doi: 10.1016/j.jclinepi.2007.04.012.

18. Tavakol M, Dennick R. Making sense of Cronbach's alpha. International journal of medical education. 2011;2: 53-55. doi: 10.5116/ijme.4dfb.8dfd.

19. Smith C, Raine K, Anderson D, et al. A preliminary examination of organizational capacity for heart health promotion in Alberta's regional health authorities. Promot Educ. 2001;Suppl 1: 40-43.

20. Barrett L, Plotnikoff RC, Raine K, Anderson D. Development of measures of organizational leadership for health promotion. Health Educ Behav. 2005;32(2):195-207. doi: 10.1177/1090 198104271970 .

21. Anderson D, Plotnikoff RC, Raine K, Cook K, Smith C, Barrett L. Towards the development of scales to measure 'will' to promote heart health within health organizations in Canada. Health Promot Int. 2004;19(4):471481. doi: 10.1093/heapro/dah409.

22. Barnes JD, Tremblay MS. Changes in indicators of child and youth physical activity in Canada, 2005-2016. Revue canadienne de santé publique. 2017; 107(6):e586-e589.

23. Faulkner G, Yun L, Tremblay MS, Spence JC. Exploration de l'impact du « nouveau » ParticipACTION : aperçu et présentation du numéro spécial. Promotion de la santé et prévention des maladies chroniques au Canada. 2018;38(4):173-82.

24. Faulkner G, McCloy C, Plotnikoff RC, Tremblay MS. Relaunching a national social marketing campaign: expectations and challenges for the "new" ParticipACTION. Health Promot Pract. 2011;12 (4):569-576. doi: 10.1177/1524 839909349180 .

25. Spence JC, Faulkner G, Costas Bradstreet C, Duggan M, Tremblay MS. Active Canada 20/20: a physical activity plan for Canada. Revue canadienne de santé publique. 2015;106(8):e470-473.
26. Marlier M, Lucidarme S, Cardon G, De Bourdeaudhuij I, Babiak K, Willem A. Capacity building through cross-sector partnerships: a multiple case study of a sport program in disadvantaged communities in Belgium. BMC Public Health. 2015;15:1306. doi: 10.1186 /s12889-015-2605-5. 